\title{
Delay-Dependent Static Output Feedback Control for Singular Time-delay Systems
}

\author{
Cuihong Wang ${ }^{1}$ Tianmin Huang ${ }^{1}$ Rui Yang ${ }^{1}$ Zhiyan Chang ${ }^{2}$ \\ ${ }^{1}$ School of Electrical Engineering, Southwest Jiaotong University, Chengdu 610031, P.R. China \\ ${ }^{2}$ Intelligent Control Development Center,Southwest Jiaotong University, Chengdu 610031, P.R. China
}

\begin{abstract}
This paper investigates the problem of static output feedback stabilization for a class of uncertain linear singular time-delay systems. Firstly, based on the stability criterion for the nominal singular timedelay system, a sufficient condition for the existence of the static output feedback controller in terms of linear matrix inequality (LMI) with linear matrix equality (LME) constraint is established, which ensures that the resulting closed-loop system is regular, impulse free and asymptotically stable. Then, by using matrix orthogonal complement, we furthermore formulate the LMI with LME constraint as a strict LMI without any constraints, and a parameterized representation of the static output feedback control law is given by the feasible solution of the LMI. Finally, a numerical example is presented to show the effectiveness of the proposed method.
\end{abstract}

Keywords: Singular systems, Time-delay, Output feedback, Linear matrix inequality (LMI)

\section{Introduction}

Recently, much attention has been devoted to the problems of robust stability and robust stabilization for uncertain singular systems[1][2]. Singular systems, which are also known as descriptor systems, semistate-space systems and generalized state-space systems, are dynamic systems whose behaviors are described by both differential equations (or difference equations) and algebraic equations [3]. Singular system models can preserve the structure of practical systems and have extensive applications in power systems, robotic systems and networks [4]. It should be pointed out that when the robust stability problem for singular systems is investigated, the problem of regularity and absence of impulses (for continuous systems) and causality (for discrete systems) are required to be considered simultaneously [5], while the latter two problems are not required to be considered in standard statespace systems.

On the other hand, many people have investigated the static output feedback problem of either certain or uncertain systems [6][7]. Although various approaches have been proposed, the analytical or numerical solution is still hard to get in general. The difficulty is that designing a static output feedback stabilization controller is actually equivalent to solve a set of bilinear matrix inequalities (BMIs), which are non-convex in general, and difficult to deal with. Recently, some efforts have been made to identify some special cases in which the solvability of the BMIs can be transformed into a set of linear matrix inequalities (LMIs) which can numerically be solved by LMI toolbox. For instant, under some sufficient conditions, it is shown that the static output sufficient conditions is solvable if the existence condition of static output feedback controller is expressed in terms of LMIs and linear matrix equality (LME) constraints [8]. To the best of our knowledge, there are only a few papers investigating the static output feedback problems for singular time-delay systems.

In the paper, the static output feedback stabilization of uncertain singular time-delay systems is investigated. Based on the stability criterion for the nominal singular time-delay system, a sufficient condition of the existence of the robust static output feedback controller is obtained in terms of LMI with LME constraint. Then using the matrix orthogonal complement method, we furthermore transfer the problem of solving the LMI with LME constraint into the problem of solving a strict LMI, which can easily be obtained feasible solution by LMI toolbox.

Notations Throughout this paper, the following notations are used. $A^{T}$ is the transpose of the matrix $A . \quad A>0(A<0)$ means $A$ is positive (negative) definite. And for a $n \times m$ full column rank matrix $A, A^{\perp}$ denotes the orthogonal complement of matrix $A . \lambda(E, A)$ represents the set $\{s \mid \operatorname{det}(s E-A)=0\}$. 


\section{Problem Formulation}

Consider the uncertain singular time-delay systems described by

$$
\begin{aligned}
E \dot{x}= & (A+\Delta A) x(t)+\left(A_{d}+\Delta A_{d}\right) x(t-d) \\
& +(B+\Delta B) u(t) \\
y(t)= & C x(t) \\
x(t)= & \varphi(t), \quad t \in[-d, 0]
\end{aligned}
$$

where $x(t) \in \Re^{n}$ is system state, $u(t) \in \Re^{m}$ is control input, $y(t) \in \Re^{m}$ is control output, $d>0$ is a constant time delay, $\varphi(t)$ is any given initial condition defined on $[-d 0]$. The matrix $E$ may be singular and we shall assume that $\operatorname{rank} E=r<n$. $A, A_{d}, B$ and $C$ are constant matrices with appropriate dimensions. In this paper, we assume that $C$ is full of rank. $\Delta A, \Delta A_{d}, \Delta B$ are unknown matrices representing the admissible uncertainties in the system matrices and can be described as the form of

$$
\left[\begin{array}{lll}
\Delta A & \Delta A_{d} & \Delta B
\end{array}\right]=H F(t)\left[\begin{array}{lll}
N_{a} & N_{d} & N_{b}
\end{array}\right]
$$

where $H, \quad N_{a}, \quad N_{d}, \quad N_{b}$ are real constant matrices with appropriate dimensions, and $F(t)$ is an unknown, real, and possibly time-varying matrix with Lebesgue measurable elements satisfying

$$
F^{T}(t) F(t) \leq I
$$

The nominal unforced counterpart of the system (1) can be written as

$$
\begin{aligned}
E \dot{x}(t) & =A x(t)+A_{d} x(t-d) \\
z(t) & =C x(t)
\end{aligned}
$$

To fascinate the following discussion, we introduce some definitions and lemmas :

\section{Definition 1[9]}

(1) The pair $(E, A)$ is said to be regular if $\operatorname{det}(s E-$ $A)$ is not identically zero.

(2) The pair $(E, A)$ is said to be impulse free if $\operatorname{deg}(\operatorname{det}(s E-A))=\operatorname{rank} E$.

(3) The pair $(E, A)$ is said to be stable if $\lambda(E, A)<$ 0 .

(4) The Pair $(E, A)$ is said to be admissible if it is regular, impulse free and stable.

Lemma 1[10] For a given scalar $d^{*}>0$, the singular time-delay system (4) is said to be regular and impulse free for any constant time delay $d$ satisfying $0 \leq d \leq d^{*}$, if the pairs $(E, A)$ and $\left(E, A+A_{d}\right)$ are regular and impulse free.
Lemma 2[10] For a given scalar $d^{*}>0$, the singular time-delay system (4) is admissible for any constant time-delay $d$ satisfying $0 \leq d \leq d^{*}$, if there exist symmetric positive definite matrices $P, Q, Z$ and matrices $S, X, Y$ such that the following linear matrix inequalities are satisfied

$$
\begin{gathered}
{\left[\begin{array}{ccc}
\Xi_{11} & \Xi_{12} & d^{*} A^{T} Z \\
* & -Q & d^{*} A_{d}^{T} Z \\
* & * & -d^{*} Z
\end{array}\right]<0} \\
{\left[\begin{array}{cc}
X & Y \\
* & Z
\end{array}\right] \geq 0}
\end{gathered}
$$

where $R \in \Re^{n \times(n-r)}$ is any full-column rank matrix satisfying $E^{T} R=0$ and

$$
\begin{aligned}
\Xi_{11}= & E^{T} P A+A^{T} P E+S R^{T} A+A^{T} R S^{T} \\
& +Y E+E^{T} Y^{T}+d^{*} X+Q \\
\Xi_{12}= & -Y E+E^{T} P A_{d}+S R^{T} A_{d} .
\end{aligned}
$$

Lemma 3[11] Given matrix $\Omega, \Gamma$ and $\Xi$ with appropriate dimensions and with $\Omega$ symmetrical, then

$$
\Omega+\Gamma F \Xi+\Xi^{T} F^{T} \Gamma^{T}<0
$$

for any $F$ satisfying $F^{T} F \leq I$, if and only if there exists a scalar $\varepsilon>0$ such that

$$
\Omega+\varepsilon^{-1} \Gamma \Gamma^{T}+\varepsilon \Xi^{T} \Xi<0 .
$$

Lemma 4[8] Suppose $D \in \Re^{n \times q}$ and $G \in \Re^{n \times q}$ are full column rank. Then there exists a $n \times n$ positive definite matrix $P$ such that $P D=G$ if and only if $D^{T} G=G^{T} D>0$. Furthermore, all solutions of $P D=G$ can be expressed as

$$
P=G\left(D^{T} G\right)^{-1} G^{T}+D^{\perp^{T}} X D^{\perp}
$$

where $X \in \Re^{(n-q) \times(n-q)}$ is an arbitrary positive definite matrix.

\section{Main Results}

In this section, we investigate the static output feedback control problem for singular time-delay system (1). To this end, we consider the following state feedback controller

$$
u(t)=K x(t)
$$

Applying this controller to the singular time-delay system (1) with $\Delta A=0, \Delta B=0$ and $\Delta A_{d}=0$ results in the closed-loop system as

$$
E \dot{x}(t)=(A+B K C) x(t)+A_{d} x(t-d)
$$


Then, the static output feedback control problem to be addressed is to design a output feedback controller (9) such that the closed-loop system (10) is admissible.

By resorting to Lemma 2, we have the solution to the static output feedback control problem as follows.

Theorem 1 For given scalars $d^{*}>0$ and $\delta_{1}, \delta_{2}$, the closed-loop singular time-delay system (10) is admissible for any constant time-delay $d$ satisfying $0 \leq d \leq d^{*}$, if there exist symmetric positive-definite matrices $P_{1}, Q_{1}, Z_{1}$, matrices $T, S_{1}, X_{1}, X_{2}, Y_{1}, Y_{2}, N$ and nonsingular matrix $M$ such that the linear matrix inequalities (11) and(12) with linear matrix equality (13) hold

$$
\begin{gathered}
{\left[\begin{array}{ccc}
\Xi_{11} & \Xi_{12} & -Y_{1} E^{T}+\delta_{1} T^{T} A_{d}^{T} \\
* & \Xi_{22} & -Y_{2} E^{T}+\delta_{2} T^{T} A_{d}^{T} \\
* & * & -Q_{1}
\end{array}\right]<0} \\
{\left[\begin{array}{ccc}
X_{1} & X_{2} & Y_{1} \\
* & X_{3} & Y_{2} \\
* & * & Z_{1}
\end{array}\right] \geq 0} \\
M C=C T
\end{gathered}
$$

then, a suitable static output feedback control law is given as

$$
u(t)=K y(t)=N M^{-1} y(t)
$$

where $R_{1} \in \Re^{n \times(n-r)}$ is any full column rank matrix satisfying $E R_{1}=0$ and

$$
\begin{aligned}
\Xi_{11}= & \delta_{1}(A T+B N C)+\delta_{1}(A T+B N C)^{T} \\
& +Y_{1} E^{T}+E Y_{1}^{T}+d^{*} X_{1}+Q \\
\Xi_{12}= & E P+S_{1} R_{1}^{T}-\delta_{1} T^{T}+\delta_{2}(A T+B N C) \\
& +E Y_{2}^{T}+d^{*} X_{2} \\
\Xi_{22}= & -\delta_{2}\left(T+T^{T}\right)+d^{*} X_{3}+d^{*} Z_{1}
\end{aligned}
$$

Proof Following the similar philosophy as that in [12], we represent the system (10) to the following equivalent form

$$
\begin{gathered}
{\left[\begin{array}{cc}
E & 0 \\
0 & 0
\end{array}\right]\left[\begin{array}{c}
\dot{x}(t) \\
\dot{z}(t)
\end{array}\right]=\left[\begin{array}{cc}
0 & I \\
\tilde{A} & -I
\end{array}\right]\left[\begin{array}{l}
x(t) \\
z(t)
\end{array}\right]} \\
+\left[\begin{array}{cc}
0 & 0 \\
A_{d} & 0
\end{array}\right]\left[\begin{array}{l}
x(t-d) \\
z(t-d)
\end{array}\right]
\end{gathered}
$$

where $z(t)=E \dot{x}(t), \tilde{A}=A+B K C$. Then, by Lemma 2 , it is easy to see that the system (14) is admissible for $0 \leq d \leq d^{*}$, if there exist symmetric positive definite matrices $P, Q, Z$ and matrices $X$, $Y$ satisfying (6) and (7) where $E$ is replaced by $\left[\begin{array}{cc}E & 0 \\ 0 & 0\end{array}\right], A$ by $\left[\begin{array}{cc}0 & I \\ A & -I\end{array}\right]$ and $A_{d}$ by $\left[\begin{array}{cc}0 & 0 \\ A_{d} & 0\end{array}\right]$.
As a particular case, we set

$$
\begin{array}{cc}
P=\left[\begin{array}{cc}
P_{1} & 0 \\
0 & \varepsilon I
\end{array}\right], & R=\left[\begin{array}{cc}
R_{1} & 0 \\
0 & P_{3}
\end{array}\right] \\
S=\left[\begin{array}{cc}
S_{1} & S_{2} \\
0 & I
\end{array}\right], & Q=\left[\begin{array}{cc}
Q_{1} & 0 \\
0 & \varepsilon I
\end{array}\right] \\
X=\left[\begin{array}{cc}
X_{1} & X_{2} \\
* & X_{3}
\end{array}\right], & Y=\left[\begin{array}{cc}
Y_{1} & 0 \\
Y_{2} & 0
\end{array}\right], \\
Z=\left[\begin{array}{cc}
Z_{1} & 0 \\
0 & \varepsilon I
\end{array}\right] &
\end{array}
$$

where $P_{1}, P_{3} \in \Re^{n \times n}$ are nonsingular matrices with $P_{1}$ symmetric and positive-definite, $R_{1} \in \Re^{n \times(n-r)}$ satisfies $E^{T} R_{1}=0$ and rank $R_{1}=n-r, S_{1} \in$ $\Re^{n \times(n-r)}, S_{2} \in \Re^{n \times n}, \varepsilon>0$. It is obvious that $\left[\begin{array}{cc}E & 0 \\ 0 & 0\end{array}\right]^{T} R=0$ and $R \in \Re^{2 n \times(2 n-r)}$ is with full column rank. By denoting $P_{2}=P_{3} S_{2}^{T}$ and letting $\varepsilon \rightarrow 0^{+}$, we obtain the following linear matrix inequalities

$$
\begin{gathered}
{\left[\begin{array}{ccc}
\Xi_{11} & \Xi_{12} & -Y_{1} E+P_{2}^{T} A_{d} \\
* & \Xi_{22} & -Y_{2} E+P_{3}^{T} A_{d} \\
* & * & -Q_{1}
\end{array}\right]<0} \\
{\left[\begin{array}{ccc}
X_{1} & X_{2} & Y_{1} \\
* & X_{3} & Y_{2} \\
* & * & Z_{1}
\end{array}\right] \geq 0}
\end{gathered}
$$

where

$$
\begin{aligned}
\Xi_{11}= & P_{2}^{T}(A T+B K C)+(A T+B K C)^{T} P_{2} \\
& +Y_{1} E^{T}+E Y_{1}^{T}+d^{*} X_{1}+Q \\
\Xi_{12}= & E^{T} P+S_{1} R_{1}^{T}-(A T+B K C)^{T} P_{3} \\
& +E^{T} Y_{2}^{T}+d^{*} X_{2}, \\
\Xi_{22}= & -P_{3}-P_{3}^{T}+d^{*} X_{3}+d^{*} Z_{1}
\end{aligned}
$$

Now, we consider the following singular timedelay system

$$
E^{T} \dot{\xi}(t)=(A+B K C)^{T} \xi(t)+A_{d}^{T} \xi(t-d)
$$

where $\xi(t)$ is the system state, and the other variables follow the same definitions as those in (1).

Noting the fact that $\operatorname{det}(s E-A)=\operatorname{det}\left(s E^{T}-\right.$ $\left.A^{T}\right)$, then the pair $(E, A)$ is regular and impulse free if and only if the pair $\left(E^{T}, A^{T}\right)$ is regular and impulse free, thus the system (10) is regular and impulse free if and only if the system (17) is regular and impulse free. And since the solution of $\operatorname{det}\left(s E-(A+B K)-e^{-d s} A_{d}\right)=0$ are the same as those of $\operatorname{det}\left(s E^{T}-(A+B K)^{T}-e^{-d s} A_{d}^{T}=0\right)$, the system (10) is stable if and only if the system (17) is stable. 
Therefore, as long as the regularity, absence of impulse and stability are concerned, we consider the system (17) instead of (10). Then, linear matrix inequality (11) can be obtained by replacing $E$ by $E^{T}, A$ by $(A+B K C)^{T}$, and $A_{d}$ by $A_{d}^{T}$ in (15) and setting $P_{2}=\delta_{1} T, P_{3}=\delta_{2} T, N=K M$ and $M C=$ $C T$. This completes the proof.

Remark 1 In the case of $C=I$, matrix $M$ and $T$ become the same variable, and the inequalities in Theorem 1 can reduce to the result of state feedback stabilization of singular time-delay system [10].

In the following, we investigate the robust static output feedback control problem for singular time-delay system (1).

Theorem 2 Consider the uncertain singular time-delay system (1), for a given scalar $d^{*}>0$ and $\delta_{1}, \delta_{2}$, if there exist symmetric positive-definite matrices $P_{1}, Q_{1}, Z_{1}$, matrices $T, S_{1}, X_{1}, X_{2}, Y_{1}, Y_{2}, N$, nonsingular matrix $M$ and scalars $\varepsilon_{1}>0, \varepsilon_{2}>0$ such that the linear matrix inequalities (18) and (12), (13)

$$
\left[\begin{array}{ccccc}
\Xi_{11} & \Xi_{12} & \Xi_{13} & \Xi_{14} & \delta_{1} T^{T} N_{d}^{T} \\
* & \Xi_{22} & \Xi_{23} & \Xi_{24} & \delta_{2} T^{T} N_{d}^{T} \\
* & * & \Xi_{33} & 0 & 0 \\
* & * & * & -\varepsilon_{2} I & 0 \\
* & * & * & * & -\varepsilon_{2} I
\end{array}\right]<0
$$

then, we can consider a suitable static output feedback control law of the form

$$
u(t)=K y(t)=N M^{-1} y(t)
$$

such that the resultant closed-loop system is admissible for all admissible uncertainties and for all constant time-delay $d$ satisfying $0 \leq d \leq d^{*}$, where $R \in \Re^{n \times(n-r)}$ is any matrix with full column rank and satisfies $E R=0$ and

$$
\begin{aligned}
\Xi_{11}= & \delta_{1}(A T+B N C)+\delta_{1}(A T+B N C)^{T} \\
& +Y_{1} E^{T}+E Y_{1}^{T}+d^{*} X_{1}+Q+\varepsilon_{1} H H^{T}, \\
\Xi_{12}= & E P+S_{1} R_{1}^{T}-\delta_{1} T^{T}+\delta_{2}(A T+B N C) \\
& +E Y_{2}^{T}+d^{*} X_{2}, \\
\Xi_{22}= & -\delta_{2}\left(T+T^{T}\right)+d^{*} X_{3}+d^{*} Z_{1} \\
\Xi_{13}= & -Y_{1} E^{T}+\delta_{1} T^{T} A_{d}^{T} \\
\Xi_{23}= & -Y_{2} E^{T}+\delta_{2} T^{T} A_{d}^{T} \\
\Xi_{33}= & -Q_{1}+\varepsilon_{2} H H^{T} \\
\Xi_{14}= & \delta_{1}\left(N_{a} T+N_{b} N C\right)^{T} \\
\Xi_{24}= & \delta_{2}\left(N_{a} T+N_{b} N C\right)^{T}
\end{aligned}
$$

Proof Replacing $A$ by $A+H F(t) N_{a}, A_{d}$ by $A_{d}+H F(t) N_{d}$ and $B$ by $B+H F(t) N_{b}$ in (11) results in the following inequality

$$
\begin{gathered}
\Lambda+\left[\begin{array}{lll}
H^{T} & 0 & 0
\end{array}\right]^{T} F(t) \Psi+\Psi^{T} F^{T}(t)\left[\begin{array}{lll}
H^{T} & 0 & 0
\end{array}\right] \\
+\left[\begin{array}{lll}
0 & 0 & H^{T}
\end{array}\right]^{T} F(t) \Phi+\Phi^{T} F^{T}(t)\left[\begin{array}{lll}
0 & 0 & H^{T}
\end{array}\right]<0
\end{gathered}
$$

where $\Lambda$ is equivalent to the left side of (11) and

$$
\begin{gathered}
\Psi=\left[\begin{array}{ccc}
\delta_{1}\left(N_{a} T+N_{b} N C\right)^{T} & \delta_{1}\left(N_{a} T+N_{b} N C\right)^{T} & 0
\end{array}\right] \\
\Phi=\left[\begin{array}{lll}
\delta_{1} N_{d} T & \delta_{2} N_{d} T & 0
\end{array}\right]
\end{gathered}
$$

By using Lemma 3, it can be shown that (20) holds for any $F(t)$ satisfying (3), if and only if there exist scalars $\varepsilon_{1}>0$ and $\varepsilon_{2}>0$ such that

$$
\begin{gathered}
\Lambda+\varepsilon_{1}\left[\begin{array}{lll}
H^{T} & 0 & 0
\end{array}\right]^{T}\left[\begin{array}{lll}
H^{T} & 0 & 0
\end{array}\right]+\varepsilon_{1}^{-1} \Psi^{T} \Psi \\
+\varepsilon_{2}\left[\begin{array}{lll}
0 & 0 & H^{T}
\end{array}\right]^{T}\left[\begin{array}{lll}
0 & 0 & H^{T}
\end{array}\right]+\varepsilon_{2}^{-1} \Phi^{T} \Phi<0
\end{gathered}
$$

which, by Shur Complement, is equivalent to (18). This completes the proof.

Remark 2 It should be pointed that the obtained conditions in Theorem 1 and Theorem 2 are not given in term of strict LMI, but in LMI with LME constraint, which is not easily solved in numerical method. Following the similar philosophy as that in [8], we formulate the problem of solving the LMI with LME constraint into the problem of solving a strict LMI.

Theorem 3 The linear matrix inequalities (18), (12) with linear matrix equality (13) is solvable, if there exists symmetric positive definite matrices $P_{1}, Q_{1}, Z_{1}, U, V$, matrices $S_{1}, X_{1}, X_{2}, X_{2}, Y_{1}, Y_{2}, N$ and scalars $\varepsilon_{1}, \varepsilon_{2}$ satisfying (12) and the following strict linear inequality

$$
\left[\begin{array}{ccccc}
\Pi_{11} & \Pi_{12} & \Pi_{13} & \Pi_{14} & \Pi_{15} \\
* & \Pi_{22} & \Pi_{23} & \Pi_{24} & \Pi_{25} \\
* & * & \Pi_{33} & 0 & 0 \\
* & * & * & -\varepsilon_{2} I & 0 \\
* & * & * & * & -\varepsilon_{2} I
\end{array}\right]<0
$$

then, we can consider a suitable static output feedback control law of the form

$$
u(t)=K y(t)=-N C C^{T} V^{-1} y(t)
$$


where $C_{0}=C^{T}\left(C C^{T}\right)^{-1}, \Upsilon=C^{T^{\perp^{T}}} U C^{T^{\perp}}$ and

$$
\begin{aligned}
\Pi_{11}= & \delta_{1}\left(A C_{0} V^{T} C_{0}^{T}+C_{0} V C_{0}^{T} A^{T}+A \Upsilon^{T}\right. \\
& \left.+\Upsilon A^{T}+B N C+C^{T} N^{T} B^{T}\right)+Q_{1} \\
& +Y_{1} E+E Y^{T}+d^{*} X_{1}+\varepsilon_{1} H H^{T} \\
\Pi_{12}= & \left(-\delta_{1} I+\delta_{2} A\right)\left(C_{0} V C_{0}^{T}+\Upsilon^{T}\right) \\
& +B N C+E Y_{2}^{T}+d^{*} X_{2}+E P+S_{1} R_{1}^{T} \\
\Pi_{22}= & -2 \delta_{2}\left(C_{0} V^{T} C_{0}^{T}+\Upsilon^{T}\right)+d^{*} X_{3}+d^{*} Z_{1} \\
\Pi_{13}= & -Y_{1} E^{T}+\delta_{1}\left(C_{0} V C_{0}^{T} A_{d}^{T}+\Upsilon A_{d}^{T}\right) \\
\Pi_{23}= & -Y_{2} E^{T}+\delta_{2}\left(C_{0} V C_{0}^{T} A_{d}^{T}+\Upsilon A_{d}^{T}\right) \\
\Pi_{14}= & \delta_{1}\left(C_{0} V C_{0}^{T} N_{a}^{T}+\Upsilon N_{a}^{T}+\left(N_{b} N C\right)^{T}\right) \\
\Pi_{24}= & \delta_{2}\left(C_{0} V C_{0}^{T} N_{a}^{T}+\Upsilon N_{a}^{T}+\left(N_{b} N C\right)^{T}\right) \\
\Pi_{15}= & \delta_{1}\left(C_{0} V C_{0}^{T} N_{d}^{T}+\Upsilon N_{d}^{T}\right) \\
\Pi_{25}= & \delta_{2}\left(C_{0} V C_{0}^{T} N_{d}^{T}+\Upsilon N_{d}^{T}\right)
\end{aligned}
$$

Proof Suppose that there exist positive definite matrices $P_{1}, Q_{1}, Z_{1}, U, V$, matrices $S_{1}, X_{1}, X_{2}, X_{2}, Y_{1}, Y_{2}, N$ and scalars $\varepsilon_{1}, \varepsilon_{2}$ satisfying (22). Let $M=V\left(C C^{T}\right)^{-1} \mathrm{M}$, then we have

$$
\begin{aligned}
C_{0} V C_{0}^{T} & =C_{0} M C \\
C C^{T} M^{T} & =M C C^{T}>0
\end{aligned}
$$

From (25) and Lemma 4, we can see that $M C=$ $C T$ has a positive definite solution

$$
T=\left(C_{0} M C\right)^{T}+\Upsilon
$$

Substituting (25) and (26) into (22) leads to (18). Thus, the linear matrix inequalities (18) and (12) with linear matrix equality (13) are solvable. This completes the proof.

Remark 3 Since $C$ is full row rank, $M=$ $V\left(C C^{T}\right)^{-1}$ is well defined.

\section{Numerical example}

In this section, we give an example to demonstrate the effectiveness of the proposed method.

Consider an uncertain singular time-delay system in (1) with parameters as follows:

$$
\begin{aligned}
& E=\left[\begin{array}{ll}
1 & 0 \\
0 & 0
\end{array}\right], \quad A=\left[\begin{array}{cc}
1 & 1 \\
-1 & -2
\end{array}\right], \quad B=\left[\begin{array}{l}
2 \\
1
\end{array}\right], \\
& A_{d}=\left[\begin{array}{cc}
0 & 0 \\
0.1 & 0.1
\end{array}\right], N_{d}=\left[\begin{array}{ll}
0 & 0 \\
0 & 0 \\
1 & 1
\end{array}\right], N_{b}=\left[\begin{array}{l}
0 \\
1 \\
0
\end{array}\right],
\end{aligned}
$$

$$
\begin{aligned}
N_{a} & =\left[\begin{array}{ll}
1 & 1 \\
0 & 0 \\
0 & 0
\end{array}\right], \quad H=\left[\begin{array}{cc}
0.1 & 0 \\
0 & 0.1 \\
0.1 & 0
\end{array}\right]^{T}, \\
C & =\left[\begin{array}{ll}
1 & 1
\end{array}\right]
\end{aligned}
$$

Now, to solve the static output feedback control problem, we choose $R=\left[\begin{array}{ll}0 & 1\end{array}\right]^{T}, \delta_{1}=6$ and $\delta_{2}=8$, then it can be checked that

$$
\begin{aligned}
P_{1} & =\left[\begin{array}{cc}
17.5283 & -0.0000 \\
-0.0000 & 26.0133
\end{array}\right], \\
Q_{1} & =\left[\begin{array}{cc}
0.5458 & -0.0012 \\
-0.0012 & 28.7737
\end{array}\right], \\
X_{1} & =\left[\begin{array}{cc}
0.0675 & -0.0509 \\
-0.0509 & 0.0545
\end{array}\right], \\
X_{2} & =\left[\begin{array}{cc}
0.0774 & -0.0803 \\
-0.0803 & 0.0825
\end{array}\right], \\
X_{3} & =\left[\begin{array}{cc}
0.1251 & -0.1283 \\
-0.1283 & 0.1345
\end{array}\right], \\
Y_{1} & =\left[\begin{array}{cc}
-0.0044 & 0.0035 \\
0.0035 & -0.0034
\end{array}\right], \\
Y_{2} & =\left[\begin{array}{cc}
-0.0047 & 0.0046 \\
0.0046 & -0.0044
\end{array}\right], \\
S_{1} & =\left[\begin{array}{cc}
-5.5175 \\
43.9842
\end{array}\right], \\
V & =2.6188, \\
N & =-1.2755, \\
U & =13.4155, \\
\varepsilon_{1} & =36.5030, \\
\varepsilon_{2} & =26.1566
\end{aligned}
$$

satisfy the linear matrix inequality (22), Therefore, by Theorem 3, we have that the static output feedback control problem is solvable, and a desired static output feedback control law can be chosen as

$$
u(t)=-1.2755 y(t)
$$

\section{Conclusions}

The problem of static output feedback stabilization for uncertain singular systems with time-delay in state is investigated in this paper. A delaydependent sufficient condition of existence of the static output feedback controller is obtained, which ensure the closed-loop system is admissible. In addition, the obtained condition for the existence of admissible controller is not expressed in terms of a strict LMI, so the matrix orthogonal complement has been explored to solve the non-convex problem. Finally, a numerical example is provided to show the applicability of the developed results. 


\section{References}

[1] J. Feng, S. Zhu and Z. Cheng, Guaranteed cost control of linear uncertain singular time-delay system, proceedings of the $41^{\text {th }}$ IEEE Conference and Decision and Control, pp: 1802-1807, 2002.

[2] X. Ji, H. Su and J. Chu, Delay-dependent robust stability of uncertain discrete singular time-delay systems, proceedings of the 2006 the American Control Conference,pp: 3843-3848, 2006.

[3] L. Dai. Singular control systems, SpringerVerlag, Berlin: Germany 1989.

[4] F.L. Lewis, A survey of linear singular systems, Circuits Systems and Signal Processing, 5: 336, 1986.

[5] S. Xu, P.V. Dooren, R. Stefan, et al, Robust stability and stabilization for singular systems with state delay and parameter uncertainty discrete singular time-delay systems, IEEE Trans. Automat. Contr, 47:1122-1128, 2002.

[6] A.R.C. Crusius and A. Trofino, A convex approach to the output feedback stabilization problem, proceedings of the 1997 the American Control Conference, pp: 2282-2283, 1997.

[7] Y. He and Q. Wang, An improved ILMI method for static output feedback control with application to multivariable PID control, IEEE Trans. Automat. Contr, 51: 1678-1683, 2006.

[8] J. Wang and J. Zhang, An LMI approach to static output feedback stabilization of linear systems, Control Theory and Application, 18: 843846, 2001.

[9] S. Xu and J. Lam, Lecture notes in control and information on robust control and filtering of singular system, Springer-Verlag, Berlin: Heidelberg 2006.

[10] H. Su, X. Ji and J. Chu, New results of robust quadratically stabilizing control for uncertain linear time-delay systems, International Journal of Systems Science, 26: 27-37, 2005.

[11] J. Lee, S. W. Kim and W. H. Kwon, Memoryless $H_{\infty}$ controller for state delayed system, IEEE Trans. Automat. Contr, 39: 159-162, 1994.

[12] E. Fridman and U. Shaked, A descriptor system approach to control of linear time-delay systems, IEEE Trans. Automat. Contr 47: 253$270,2002$. 